\title{
The oddity preference effect and the concept of difference in pigeons
}

\author{
Thomas A. Daniel ${ }^{1,2}$ • Anthony A. Wright ${ }^{3}$ - Jeffrey S. Katz ${ }^{1}$
}

Published online: 8 April 2016

(C) Psychonomic Society, Inc. 2016

\begin{abstract}
Previous work in discrimination learning has shown that nonmatching (oddity) tasks are learned faster and more accurately than comparable matching tasks. This learning advantage has been coined the oddity preference effect (Wright \& Delius in Journal of Experimental Psychology: Animal Behavior Processes, 31, 425-432. doi:10.1037/ 0097-7403.31.4.425,2005). Pigeons trained in a nonmatching task, following training in a same/different (S/D) task, learned the abstract concept of difference (Daniel et al., in Animal Cognition, 18(4), 831-837, 2015), but they did not show the expected faster acquisition or high levels of transfer from the oddity preference effect. In the present study, experimentally naïve pigeons were trained in an identical nonmatching task to examine whether they would show the oddity preference effect on abstract-concept learning. These experimentally naïve pigeons did show an oddity preference effect; their transfer to novel configurations was above chance with the initial (smallest) set size (3-item set) and was substantially more accurate than novel transfer in similar match-tosample (MTS) or S/D tasks (Bodily et al., in Journal of Experimental Psychology: Animal Behavior Processes, 34, 178-184. doi:10.1037/0097-7403.34.1.178, 2008; Katz \& Wright in Journal of Experimental Psychology: Animal Behavior Processes, 32, 80-86. doi:10.1037/0097-7403.32.
\end{abstract}

Thomas A. Daniel tdaniel16@gmail.com

1 Auburn University, Auburn, AL, USA

2 College of William \& Mary, P.O. Box 8795, Williamsburg, VA 23187, USA

3 University of Texas Health Science Center at Houston, Houston, TX, USA
1.80, 2006). As the number exemplars in the training set increased, transfer to novel configurations increased and reached equivalence to trained-stimulus performance with a 24-item set. Despite this transfer being equal to baseline performance with a 24-item set, subsequent transfers following training with larger set sizes declined before eventually rising again to baseline performance. This unusual set-size function (with inflection points at the 24- and 96-set sizes) suggests that these pigeons may have combined item-specific and relational learning strategies with differing emphasis as they acquired the abstract concept.

Keywords Concept learning · Discrimination · Matching · Pigeon $\cdot$ Nonmatching

Concepts aid the organism's efficient processing of its environment. Abstract concepts are defined by particular relationships between two or more stimuli that transcend physical characteristics of individual stimuli and provide the basis for transfer to novel stimuli and contexts. Animals may use an abstract concept to solve a task that is new and not previously experienced or to solve a task where instances of rote memorization or generalization are impossible (Wasserman, DeVolder, \& Coppage, 1992; Wright \& Katz, 2007). For example, a bird may judge that a new insect is the same as, different than, or smaller than a previously eaten insect. This differs from natural concepts, also referred to as categories (Thompson, 1995), which rely on a commonality of individual physical features. For example, a bird that eats insects does not need to remember that each individual species of insect is edible; instead, individual species can be represented by an "insect" category based on features like color, shape, or movement. Unlike abstract concepts, natural concepts rely on stimulus generalization to transfer behavior to novel stimuli. 
Abstract concepts were previously claimed to be a behavior that relied exclusively on the capacity for language (Premack, 1978,1983 ) because early studies with pigeons failed to show conclusive evidence for abstract-concept learning (Berryman, Cumming, Cohen, \& Johnson, 1965; Cumming \& Berryman, 1961; Farthing \& Opuda, 1974). These studies used limited training sets, which have since been shown to promote itemspecific learning strategies (Bodily, Katz, \& Wright, 2008; Daniel, Wright, \& Katz, 2015; Katz \& Wright, 2006). Such findings highlight the importance of large or expanding training sets showing that as more exemplars are used during training, the transfer more closely approaches baseline performance. Using match-to-sample (MTS) or same/different (S/D) procedures, a wide variety of species have demonstrated the ability to apply accurately abstract relational rules, including: baboons (Bovet \& Vauclair, 2001), bumblebee colonies (Brown \& Sayde, 2013), chimpanzees (Premack, 1978, 1983), dolphins (Herman, Hovancik, Gory, \& Bradshaw, 1989), humans (e.g., Bukatko \& Daehler, 2011; Hochmann, Mody, \& Carey, 2016), monkeys (e.g., Katz, Wright, \& Bachevalier, 2002; Wright, Rivera, Katz, \& Bachevalier, 2003), parrots (Pepperberg, 1987), pigeons (Wright, 1997), and sea lions (Kastak \& Schusterman, 1994).

Along with MTS and S/D, pigeons also have the capacity to learn the concept of difference using a nonmatching-to-sample (NMTS) procedure (Daniel et al., 2015). In that 2015 study, the number of stimuli used during training were systematically doubled following an accuracy criterion and novel-stimulus transfer. For example, after acquiring NMTS with a training set of three stimuli, transfer by the pigeons was tested with pairs of novel stimuli, then the set size was doubled to six stimuli, followed by a new transfer test with pairs of novel stimuli. This sequence of training, testing, and set-size expansion continued until pigeons successfully acquired NMTS with a set size of 768 stimuli. With the earlier training sets, the evidence indicated that the pigeons were using item-specific learning strategies because their novelstimulus accuracy was at or close to chance performance (50\% correct). However, as the training set size increased, so did performance on novel configurations until it was equivalent to baseline performance with trained configurations. Thus, those pigeons learned the relationship between the stimuli in the configuration and were able to apply a relational rule (i.e., select the nonmatching stimulus from the configuration).

The set-size function of nonmatching (Daniel et al., 2015) was nearly identical to the set-size function of matching obtained by Bodily et al. (2008); as the number of training stimuli increases, accuracy for novel items rose from chance to baseline. This finding contradicted those from earlier studies that compared MTS and NMTS (Berryman et al., 1965; Ginsburg, 1957; Mishkin \& Delacour; 1975; Wilson, Mackintosh, \& Boakes, 1985; Zentall, Edwards, Moore, \& Hogan, 1981) where NMTS was learned much more quickly, often with an initial transfer above chance. Those studies showed an oddity preference effect (OPE), perhaps resulting from extinction of observing responses to the sample stimulus (i.e., not being followed by primary reinforcement) and only advancing the trial to the choice stimuli (Wright \& Delius, 2005). According to the argument, when the comparison array appeared, subjects were more likely to respond to the stimulus that was different from the sample because the previous response to the sample had not been rewarded. Similarly, Zentall and Hogan (1974) posited that this sample response partially extinguishes responding to the sample stimulus. This explanation was tested by reinforcing the pigeons' observing response to the sample stimulus and groups of pigeons trained in MTS and NMTS showed no differential rates of acquisition, thus eliminating the OPE (Wright \& Delius, 2005). In light of such findings, pigeons in the Daniel et al. (2015) study would have been expected to transfer above chance at the earliest set size and have acquired the task faster than their MTS counterparts (Bodily et al., 2008). But they did not. One possible explanation is that the pigeons in the Daniel et al. (2015) study had extensive learning experience in a S/D task that may have affected their susceptibility to the OPE (Schmidtke, Katz, \& Wright, 2010). If the absence of the OPE in Daniel et al. (2015) resulted from prior S/D experience, then naïve pigeons performing an identical task should show an OPE with increased acquisition and better novel-stimulus transfer.

The purpose of the current study was to determine whether or not experimentally naïve pigeons would learn NMTS differently, perhaps more readily, than those with prior S/D experience, or others trained and tested in the MTS task.

\section{Method}

All methods used in this study matched those used in the Daniel et al. (2015) study for training and testing in the NMTS task, including the same training stimuli, apparatus, sample FR requirement, performance criteria, transfer testing, transfer stimuli, and set-size expansions followed by transfer tests.

\section{Subjects}

Four experimentally naïve male pigeons (Columba livia), ages 6 to 12 months old, from the Palmetto Pigeon Plant, served as subjects. Subjects were maintained within $80 \%$ to $85 \%$ of their free-feeding body weight throughout the study; in the event that a subject's weight fell above or below this range for the day, it did not participate in that day's session. Subjects resided in a colony room governed by a 12-hour light/ day cycle and were housed individually with free water and grit access. 


\section{Apparatus}

Pigeons were tested using custom built wooden (35.9-cm wide $\times$ 45.7-cm deep $\times$ 51.4-cm high) testing chambers. A fan (Dayton 5C115A, Niles, IL) located in the back wall of each chamber provided ventilation and white noise. The computer detected pecks via an infrared touch screen (17-in. Unitouch, Carroll Touch, Round Rock, TX). This pressure-fit touch screen was situated within a $40.6-\mathrm{cm} \times$ $32.1-\mathrm{cm}$ cutout in the front panel that was centered $7.7 \mathrm{~cm}$ from the top of an operant chamber. A 28-V (No. 1829, Chicago Miniature, Hackensack, NJ) house light, located in the center of the ceiling, illuminated the chamber during intertrial intervals (ITI). A custom hopper containing mixed grain was accessed by pigeons through an opening $(5.1 \times 5.7 \mathrm{~cm})$ centered in the front panel $3.8 \mathrm{~cm}$ above the chamber floor.

Custom software written with Visual Basic 6.0 on a Dell Optiplex GX110 recorded and controlled all events in the operant chamber. A video card controlled graphics generated by the computer while a computer-controlled relay interface (Model No. PI0-12, Metrabyte, Taunton, MA) maintained operation of the grain-hopper and the lights to both the hopper and the chamber.

\section{Stimuli}

Visual stimuli were computer-created, color cartoon JPEG images that were $2.5-\mathrm{cm}$ high $\times 3-\mathrm{cm}$ wide at $28 \mathrm{pixel} / \mathrm{cm}$ (cf. Katz, Bodily, \& Wright, 2008, Figure 2). Stimuli were arranged in the display such that the sample and comparisons formed a triangle $(8-\mathrm{cm}$ high $\times 19$-cm wide) with the comparison stimuli symmetrically placed around the sample. Each sample stimulus appeared centered horizontally at approximately $8 \mathrm{~cm}$ above the bottom of the display. Left- and right-comparison stimuli appeared $4 \mathrm{~cm}$ above the bottom of the monitor, with their outermost edges positioned $9 \mathrm{~cm}$ from the display's edge.

\section{Pretraining}

Subjects learned to peck at the computer monitor via autoshaping. Pecking was autoshaped using one of three cartoon stimuli (apple, grape, or duck) in two possible stimuli locations: left comparison and right comparison. After displaying the cartoon for $10 \mathrm{~s}$, access to mixed grain was provided for $5 \mathrm{~s}$. This grain access was followed by a 50-s ITI before another stimulus was displayed. Pecking at the displayed stimulus terminated the cartoon and raised the hopper. This sequence was repeated for 96 trials per autoshaping session. Subjects continued autoshaping until at least $80 \%$ of the 10 -s stimulus presentations received a response. After subjects responded reliably, training began in a response-dependent session that required one peck to a stimulus (FR 1) to provide reinforcement followed by a 15-s ITI. All other details were the same as the autoshaping sessions. When subjects completed this session within 2 hours, they advanced to the training phase of the study.

\section{Training}

Daily sessions were conducted 5 to 7 days a week. Each session consisted of 96 trials, with half of the trials rewarding responses to the left-choice stimulus and half rewarding response to the right-choice stimulus. Pigeons were initially trained in NMTS with the same three stimuli used during autoshaping. All trials began with a sample stimulus displayed on the monitor. Pigeons observed a sample stimulus by making 10 responses to the image; this FR requirement began with one peck but was systematically increased over seven sessions to 10 pecks. After subjects performed the response requirement, two comparison stimuli were presented; one comparison stimulus matched the sample, and the other was randomly selected from the remaining training set. A response to the nonmatching comparison resulted in grain reinforcement with the nonmatching comparison displayed for $4 \mathrm{~s}$. Grain access was between 2 and 3.5 s of mixed grain depending on the pigeon's body weight prior to the session. A response to the matching comparison resulted in an 8-s timeout where the sample and matching comparison stimulus were removed. All trials were followed by a 15-s ITI whether the response was correct or incorrect. With a set size of three stimuli, there were 12 possible configurations; these configurations appeared eight times per 96-trial session. Stimuli were counterbalanced to ensure that a configuration would not directly repeat itself on the next trial. Correct response locations (left or right) were also counterbalanced so that an equal number of correct left and right responses occurred in any given session.

Training continued until a pigeon reached $85 \%$ accuracy across two consecutive sessions with a correction procedure (CP). The CP forced subjects to repeat any incorrect trials until a correct response was made, but only the first response to each trial was counted and computed for accuracy. After this performance-based criterion was met, the subjects were required to perform $85 \%$ accuracy on one session without the $\mathrm{CP}$ before proceeding to transfer testing.

\section{Transfer testing}

The testing phase was composed of four 96-trial sessions and began on the next daily session after a pigeon achieved criteria-based performance. Within each testing session, 12 novel stimulus trials were randomly mixed with 84 training trials, resulting in exposure to 24 novel stimuli each testing session and a total of 96 ( 24 stimuli $\times 4$ sessions) novel stimuli 
per testing phase. Stimuli presented in these testing configurations were unique and never viewed by the subjects prior to the transfer test. No testing trial appeared within the first or last eight trials of any transfer session; at least five trials separated any testing trial from another. Responses on testing trials were reinforced similar to training trials; a correct response resulted in grain access, and an incorrect response resulted in an 8-s timeout period. Testing trials and sessions were also counterbalanced for left and right responses using the same specifications as those during training.

\section{Set-size expansion}

Following transfer testing, an equal number of new training stimuli were added to the previous training set. The number of images used in training increased from 3 to $6,12,24,48,96$, 192,384 , and 768. Pigeons were required to achieve criterion performance ( $>85 \%$ accuracy on one session without $\mathrm{CP}$ and a minimum of three sessions) with each expanded training set before transfer testing. If a pigeon performed below $75 \%$ accuracy, CP was reinstated until two consecutive sessions above $85 \%$ accuracy were obtained. Training and transfer testing followed the same structure for all set-size expansions.

\section{Results}

\section{Acquisition}

Set-size three All birds reached criterion on the initial set size in around eight sessions $(M=792$ trials, range $=672-960$ trials). Mean percentage correct between the first $(58.6 \%)$ and last $(90.6 \%)$ session of three-item training significantly increased, as confirmed by a repeated measures ANOVA, $F(1$, $3)=47.131, p<.01$.

Set-size expansion Mean number of trials to reach criterion for set sizes larger than three items varied across sessions, ranging from 288 to 792 trials. This was confirmed by a one-way repeated-measures ANOVA for set size on trials-to-acquisition, $F(8,24)=5.2, p<.01$. A trend analysis yielded a significant quadratic component, $F(1,3)=38.5, p<$ .01 . Acquisition was slowest at set sizes $3,192,384$, and 768 $(M=630)$ which did not differ significantly among them, $F(3$, $9)=2.1, p=.17$. Acquisition was fastest at set sizes 6, 12, 24, 48 , and $96(M=345.6)$, which also did not differ significantly among them, $F(4,12)=1.6, p=.25$.

First-session of set-size expansion During acquisition, training sets were expanded and new training stimuli were introduced, resulting in trials that contained both trained and untrained stimuli. For analyses, trials were classified into one of four combinations, where the sample and/or comparison were new (untrained) stimuli or old (trained) stimuli (cf., Bodily et al., 2008; Daniel et al., 2015). When the sample was chosen from a previously trained set size, combinations were either sample trained, nonmatching trained (ST-NMT) or sample trained, nonmatching untrained (ST-NMU). When the sample was chosen from the newly added stimuli, combinations were either sample untrained, nonmatching trained (SU-NMT) or sample untrained, nonmatching untrained (SU-NMU). Accordingly, trial combinations in which all stimuli were experienced in the previous training set would be classified as ST-NMT, and combinations in which all stimuli were first introduced in the newly expanded training set would be classified as SU-NMU.

A two-way repeated-measures ANOVA of Stimulus Combination (ST-NMT, ST-NMU, SU-NMT, SU-NMU) $\times$ Set Size $(6,12,24,48,96,192,384,768)$ show differences in stimulus combination, $F(3,9)=5.8, p<.05$, no difference of set size, $F(7,21)=1.015, p=.449$, and an interaction, $F(21$, $63)=2.5, p<.01$. This interaction was due to performance at set sizes 6,12 , and 24 , when the nonmatching choice stimulus was previously trained (ST-NMT, SU-NMT; $M=92.48$ ) the pigeons selected it more than when it was untrained (SU-NMU, ST-NMU; $M=76.27$ ), paired-sample $t$ test, $t(3)$ $=7.372, p<.01$. From set sizes 48 to 192 the pigeons were more influenced by whether the sample stimulus had been trained (ST-NMT, ST-NMU; $M=93.26$ ) than untrained (SU-NMT, SU-NMU; $M=78.42$ ), paired-sample $t$ test, $t(3)$ $=6.84, p<.01$. There were no differences between the four trial types for set sizes 384 and $768, F(3,9)=1.86, p=.21$. To emphasize the interaction, accuracy of the stimulus combinations with mixed experience (i.e., ST-NMU and SU-NMT) are shown in Fig. 1.

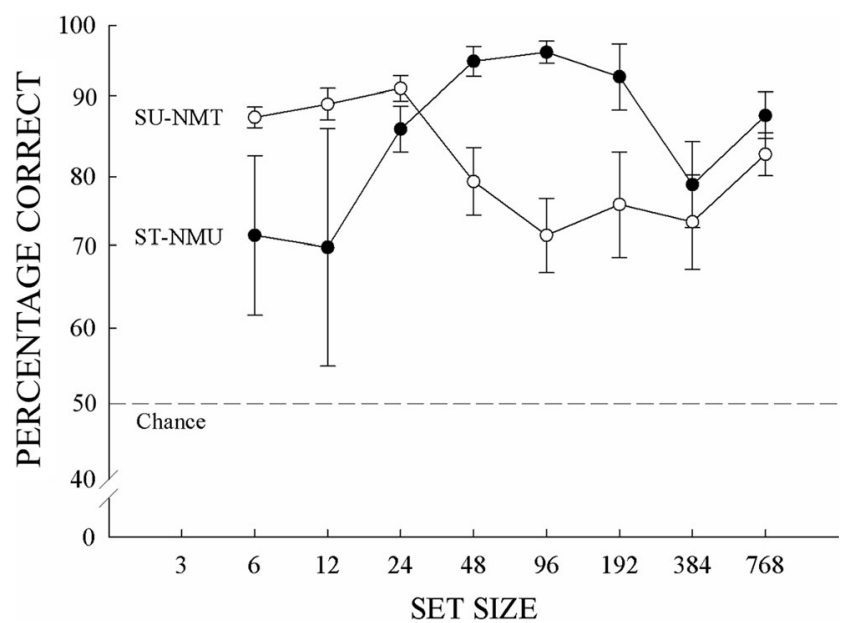

Fig. 1 Mean percentage correct on first-session acquisition across set size. Two trial types, sample-trained, nonmatching-untrained (ST-NMU, filled symbols) and sample-untrained, nonmatching-trained (SU-NMT, open symbols), are plotted across the expanding set size. The dashed line represents $50 \%$ chance accuracy. Error bars represent SEMs 


\section{Transfer}

Figure 2 shows mean baseline and transfer accuracy averaged across the four testing sessions at each training set size. Baseline accuracy on the initial set size $(M=89.21)$ did not change by the final set size $(M=88.54)$. Transfer percentage correct for the initial three-item set was $67 \%$ and significantly above chance, $t(3)=4.371, p<.05$. Transfer accuracy rose $21 \%$ from the initial three-item set size to $88 \%$ on the final 768 set size. A two-way repeated-measures ANOVA of Condition (baseline, transfer) $\times$ Set Size $(3,6,12,24,48$, $96,192,384,768)$ confirmed a difference of condition, $F(1$, $3)=95.183, p<.01$, no difference of set size, $F(8,24)=1.907$, $p=.106$, and an interaction $F(8,24)=3.368, p<.05$. This interaction was due to stable performance on baseline trials, whereas transfer performance steadily increased over set size, as confirmed by separate one-way repeated-measures ANOVAs, baseline: $F(8,24)=1.538, p=.197$; transfer: $F(8,24)=2.624, p<.05$. All pigeons showed an early increase in transfer from 3 to 24 with the highest level of transfer at the 24-item set (one pigeon showed equally high transfer at the 12-item set). A trend analysis on set size $(3,6,12,24)$ found only a significant linear component, $F(1,3)=13.765, p$ $<$.05. All pigeons showed a subsequent decrease in transfer after the 24-item set followed by another increase in transfer at the larger set sizes. A trend analysis on set size (24, 48, 96, $192,384,768$ ) found only a significant quadratic component, $F(1,3)=30.94, p<.05$. At set-sizes $24,48,192,384$, and 768, transfer was not significantly different from baseline percentage correct, paired-sample $t$ tests, $t \mathrm{~s}(3)<2.62, p>.05$.

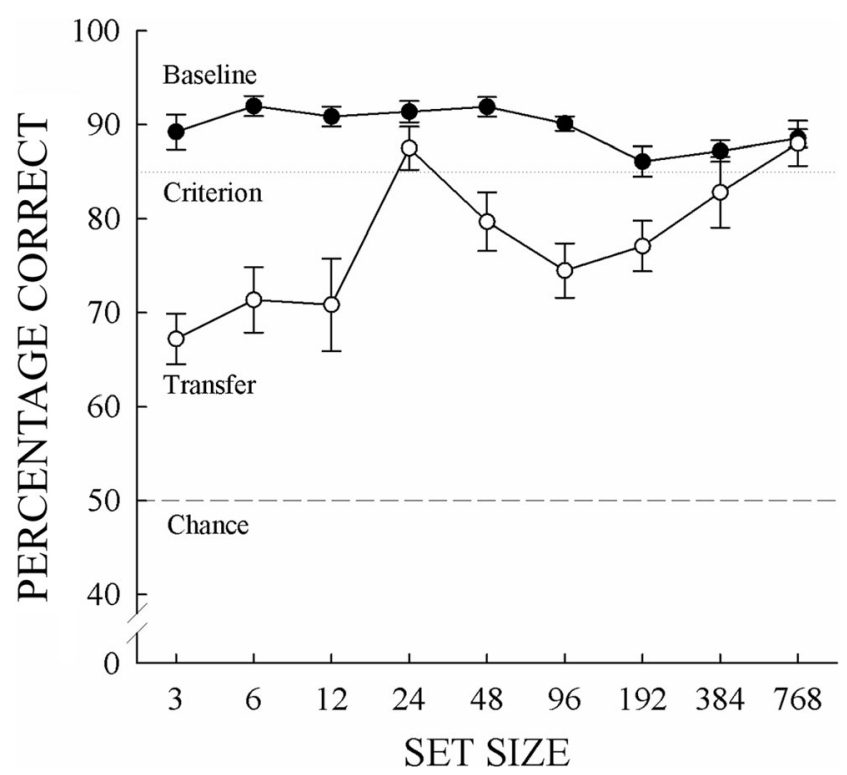

Fig. 2 Mean baseline (filled symbols) and transfer (open symbols) percentage correct across set size. The horizontal dotted line represents $85 \%$ accuracy performance criterion used during acquisition. The horizontal dashed line represents $50 \%$ chance accuracy. Error bars represent SEMs
Additionally, at every set size, transfer accuracy was significantly above chance $(50 \%)$, as confirmed by one-sample $t$ tests, $t \mathrm{~s}(3)>3.5, p \mathrm{~s}<.05$. A series of two-way repeated-measures ANOVAs of Side (left, right) $\times$ Session $(1,2,3,4)$ was conducted on transfer accuracy to test for response biases and acquisition across transfer testing for each set size. At each set size, transfer accuracy did not change across testing sessions ( $p s>.05$ ) indicating performance was constant and no side biases were detected ( $p \mathrm{~s}>.05)$.

\section{Discussion}

In this discussion, results from the group of experimentally naïve pigeons learning NMTS will be first compared to a study that was similar in many respects, except for prior S/D experience (Daniel et al., 2015). Since precisely the same NMTS procedures were used in the Daniel et al. study (same training stimuli, apparatus, sample-FR requirement, performance criteria, transfer testing, transfer stimuli, and set-size expansions), any substantial NMTS performance differences can be attributed to their prior $\mathrm{S} / \mathrm{D}$ experience for an as-direct-as-possible comparison. Additionally, a second comparison will be made to an MTS study that also shared all of those procedures listed above (except MTS instead of NMTS), and therefore also makes an as-direct-as-possible comparison.

In both the study reported here and the Daniel et al. (2015) study, novel transfer became as accurate at baseline performance by the final set-size expansion, as shown by novel transfer performance being as accurate as baseline performance, which remained constant and consistently high across the expanding set sizes. But unlike the Daniel et al. study, pigeons in the study presented here transferred above chance $(M=67 \%)$ following training with the first and smallest set size of three items, compared to $(M=55 \%)$ the Daniel et al. study. Chance, or near chance, transfer performance is typical in other concept learning tasks, such as S/D (e.g., pigeons, capuchins, rhesus monkeys; Wright \& Katz, 2006, but see Magnotti, Katz, Wright, \& Kelly, 2015, for Clark's nutcrackers). The initial above-chance accuracy shown for NMTS in the study reported here replicates the OPE found in many others studies (e.g., Ginsburg, 1957; Wilson et al., 1985; Wright \& Delius, 2005; Zentall \& Hogan, 1974) but was conspicuously absent from Daniel et al. study. As Wright and Delius (2005) demonstrated, the OPE is produced by nonreinforcement of sample responses, resulting in a greater likelihood that the comparison stimulus with no immediate history of extinction will be chosen. Thus, extinction-associated novel sample stimulus responding would also produce above-chance performance on transfer trials and the OPE. That is, on transfer trials the pigeons encounter a novel sample and do not receive reinforcement 
for pecking at it. Next, when they encounter this same stimulus as a choice item, they avoid responding to this item and choose the alternative - the nonmatching item. Therefore, when faced with novel items for the first time with no prior history of reinforcement, such an OPE strategy could be effective. This mechanism is not unlike dishabituation in a visual paired-comparison test, where newer stimuli attract attention more than familiar ones (Fagan, 1971; Pascalis, de Haan, \& Nelson, 2002).

In addition to transferring above chance at the earliest set size, pigeons in the NMTS study reported here also transferred at a level equivalent to baseline accuracy with the 24-item training set (transfer rose from $70.8 \%$ with the 12 -item set to $87.5 \%$ with the 24 -item set). By most any measure, such a level of concept learning should be evidence that the concept had been learned. Nevertheless, this evidence for concept learning was contradicted by a fall in transfer performance with further set-size expansion (transfer fell from $87.5 \%$ to $79.7 \%$ on the 48 -item set and to $74.5 \%$ with the 96 -item set).

The most likely explanation for why transfer was more accurate with the 24-item set than the next larger training sets appears to be due to the OPE and the item-specific learning strategies engendered by the OPE. Pigeons solving the task by responding to the comparison stimulus not recently extinguished (i.e., the nonmatching stimulus) can apply this strategy to novel configurations and produce only what initially appears to be full-concept learning. In addition to any OPE, pigeons begin applying the relational rule of difference (i.e., pick the stimulus that does not match the others). As the training set size increases, the difference rule and OPE inexplicably come into conflict. As the relational rule of difference gains prominence the item-specific response strategy of the OPE wanes for reasons that are not entirely clear; nevertheless, patterns of performance immediately following set-size expansion provide some insights into the interplay of these different learning types.

\section{First-session of set-size expansions}

If pigeons were using an abstract relational rule to solve the task, it should not have mattered whether the stimuli were previously used in training or were newly added. However, if pigeons learned something other than the abstract relational rule, their pattern of responses on different stimulus combinations should be indicative of what they had learned and how they were performing this NMTS task. In this light, ST-NMT trials are similar to baseline trials because the stimuli were previously used in training, and SU-NMU trials are similar to transfer trials because the stimuli were not used in any earlier set size. How pigeons respond to trial types where the training history of the stimuli is mixed (i.e., ST-NMU and
SU-NMT) is of particular interest here to look for influence of the OPE.

First-session performance analyses show that pigeons are not immune to the reinforcement history of the prior training set. During the earliest set sizes, the training history of the stimuli predicted performance on the task, but as the set size increased, differences between these trial types vanished. That is, pigeons performed equally well on all trial types regardless of the training history of the stimuli used. Initially, accuracy was highest on configurations where the nonmatching comparison was previously experienced but was not the sample (SU-NMT). This increased accuracy is accommodated by the OPE's influence on behavior; the lack of reinforcement history of the sample paired with the previously established reinforcement history of the nonmatching comparison resulted in accuracy that was higher than other trial types. This finding is in line with Wright and Delius's (2005) assertion that the OPE is created by a lack of reinforcement provided for observing the sample stimulus.

As Fig. 1 shows, pigeons responded to the mixed-training trial types differently across set size. At early set sizes, accuracy was higher when the nonmatching comparison had prior training, but after the 24-item set, this pattern reversed. This switch in responding highlights the change in item-specific learning strategies described above. The initial advantage of the OPE disappears after the 24-item set, which is the same point when all pigeons initially transferred to baseline, after which pigeons employ a new item-specific strategy that will lead toward learning the relational rule. The function of ST-NMU and SU-NMT follows the transfer accuracy across set size: as pigeons shift their responding away from the history of the sample stimulus, transfer accuracy temporarily drops. By the final set sizes, however, pigeons perform well regardless of the history of the stimuli because they are applying the abstract concept of difference.

\section{Comparison to matching and same/different}

Alone, the present study offers further evidence of abstract-concept learning in pigeons. Comparisons with MTS (Bodily et al., 2008), S/D (Katz \& Wright, 2006), and nonnaïve subjects in NMTS (Daniel et al., 2015) provide additional insights into abstract-concept learning. Figure 3 contrasts the set-size functions between the current results with those from MTS, S/D, and nonnaïve subjects in NMTS. While the increase in transfer performance is clear from the 3 to 768 set sizes, there are two important differences found between the current study and Daniel et al., MTS (Bodily et al., 2008), or S/D (Katz \& Wright, 2006). First, during testing of the smallest set size, pigeons in the current experiment transferred above chance. This finding is particularly striking as pigeons 


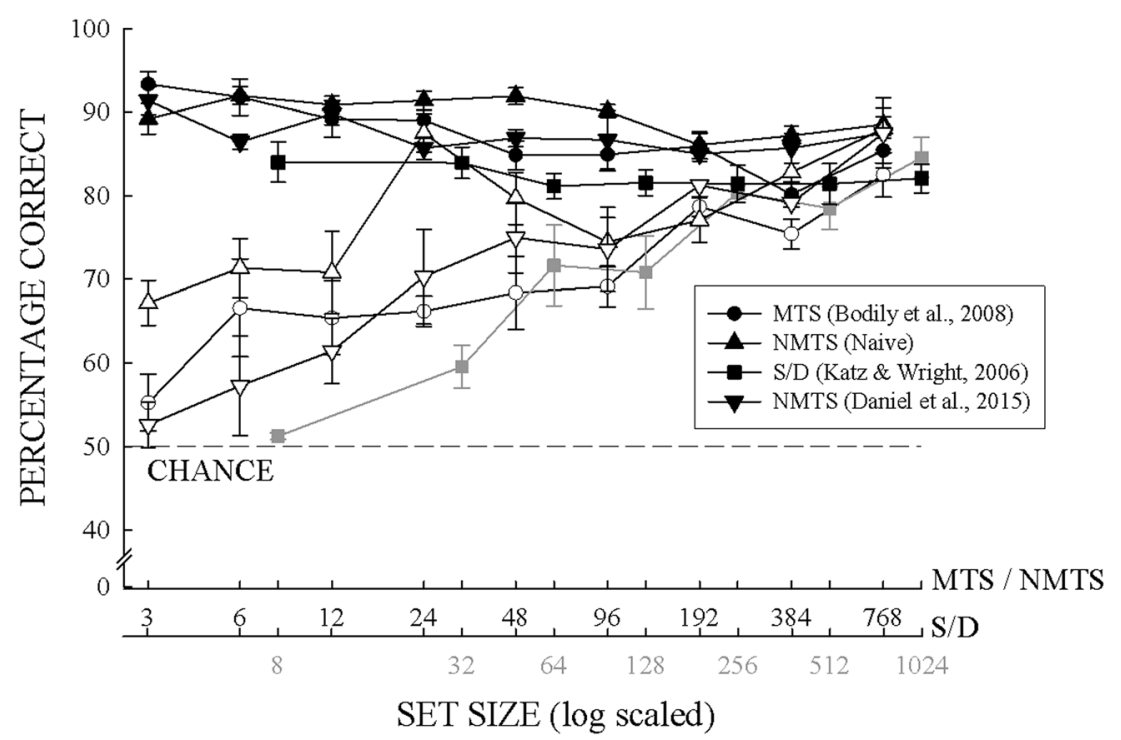

Fig. 3 Mean baseline (dark symbols) and transfer (light symbols) percentage correct across set size for MTS (Bodily et al., 2008; circles), NMTS with naïve pigeons (inverted triangles), NMTS with experienced

(or capuchin and rhesus monkeys; Katz et al., 2002; Wright et al., 2003) did not transfer above chance at the initial training set size in previous set-size expansion studies. Second, unlike previous set-size studies, transfer accuracy did not increase gradually in a linear fashion as a function of set size, as did the transfer accuracy from MTS, S/D, or even NMTS with pigeons first trained in S/D. Transfer from set-sizes 3 to 24 showed a $20.3 \%$ monotonic increase from $67.2 \%$ to $87.5 \%$, where it was both above chance and equivalent to baseline $(91.37 \%)$. Transfer performance then dropped and came back up again, forming a $U$-shaped function. These nonlinearities in the set-size function suggest changes in the basic learning strategy over set-size expansion, with those sets between 12 and 48 serving as a key transition point.

One might have predicted that the current study's pigeons would learn the task faster due to the OPE. However, these pigeons did not learn the initial three-item training set faster than naïve pigeons trained in MTS ( $M=832$ trials; Bodily et al., 2008) or nonnaïve, experienced pigeons trained in NMTS ( $M=768$; Daniel et al., 2015). While the OPE did not influence the acquisition rate of the initial training set, the OPE can be seen by comparing the first-session performance of the current study's pigeons with Daniel et al. (2015). The experimentally naïve pigeons of the current study performed more accurately on NMU trials $(72.6 \%)$ than the experienced pigeons of Daniel et al. (52.89\%) for set-sizes 6 and 12. Transfer tests complement these first-session analyses as experimentally naïve pigeons transferred above chance on the initial set sizes, and the experienced pigeons transferred at chance. pigeons (Daniel et al., 2015; triangles), and $S / D$ (Katz \& Wright, 2006; squares). The horizontal dashed line represents $50 \%$ chance accuracy. Error bars represent SEMs

The study presented in this article and the comparisons to Katz and Wright (2006) and Daniel et al. (2015) stress the importance of prior learning experience on the formation of abstract concepts. Prior to participating in NMTS, the pigeons of Daniel et al. (2015) had demonstrated full S/D concept learning (see Schmidtke et al., 2010). In this S/D task, these pigeons were required to respond whether two items in a configuration were the same or different. Accordingly, the sample in an S/D configuration cannot by itself determine whether a same or a different response will be correct; therefore, item-specific strategies like the OPE would be ineffective. These nonnaïve, experienced pigeons learning and performing in NMTS demonstrated a set-size function similar to the pigeons trained in MTS (Bodily et al., 2008) apparently because there was no OPE (see also Zentall et al., 1981, for an experienced-diminished OPE). The naïve pigeons of the present study, however, show all the hallmarks of the OPE, including the above-chance performance at the initial set size, and moreover show the evolving interplay between the OPE and relational concept learning not shown in any prior studies.

\section{Conclusions}

Across a variety of species, the relationship between increasing set size and concept learning is consistent: with initial small stimulus sets, transfer is close to chance performance, then as the number of training exemplars is gradually increased, transfer to novel stimuli also gradually increases. By contrast, experimentally naïve pigeons in NMTS transfer 
above chance, steadily increase their transfer until the 24-item set, subsequently decrease transfer at the 48 -item set, and then finally gradually increase their transfer again until full concept learning is obtained. These data and accompanying comparisons show the importance of parametric manipulation of set size. If the pigeons of the study presented here had never been tested with set sizes greater than 24 items, we might have mistakenly assumed that full concept learning was obtained with the 24-item set. These functions and comparisons highlight the contribution of the OPE, as pigeons' initial nonmatching behavior was controlled by item-specific rules (i.e., select the stimulus that was not just extinguished). However, like all other set-size functions, as the set size increased, these naïve pigeons developed abstract relational rules to solve the discrimination task (i.e., select the nonmatching stimulus). By systematically testing the pigeon's set-size function for NMTS, these studies show not only whether pigeons could learn the concept of difference, but how they learned the concept.

Acknowledgments We wish to thank Adam M. Goodman, John F. Magnotti, Andrea M. Thompkins, and Kent D. Bodily for their careful assistance in conducting this experiment. Portions of this experiment were used in Thomas A. Daniel's master's thesis.

\section{Compliance with ethical standards}

Conflict of interest statement The authors declare no competing financial interests.

Ethical standards This experiment complied with current United States law and following the relevant ethical guidelines for animal research (IACUC approved and conducted in AAALAC approved facilities).

\section{References}

Berryman, R., Cumming, W. W., Cohen, L. R., \& Johnson, D. F. (1965). Acquisition and transfer of simultaneous oddity. Psychological Reports, 17, 767-775.

Bodily, K. D., Katz, J. S., \& Wright, A. A. (2008). Matching-to-sample abstract-concept learning by pigeons. Journal of Experimental Psychology: Animal Behavior Processes, 34, 178-184. doi:10. 1037/0097-7403.34.1.178

Bovet, D., \& Vauclair, J. (2001). Judgment of conceptual identity in monkeys. Psychonomic Bulletin \& Review, 8, 470-475.

Brown, M. F., \& Sayde, J. M. (2013). Same/different discrimination by bumblebee colonies. Animal Cognition, 16, 117-125.

Bukatko, D., \& Daehler, M. W. (2011). Child development: A thematic approach. Belmont, CA: Wadsworth.

Cumming, W. W., \& Berryman, R. (1961). Some data on matching behavior in the pigeon. Journal of the Experimental Analysis of Behavior, 4, 281-284.
Daniel, T. A., Wright, A. A., \& Katz, J. S. (2015). Abstract concept learning of difference in pigeons. Animal Cognition, 18(4), 831837. doi:10.1007/s10071-015-0849-1

Fagan, J. (1971). Infant recognition memory for a series of visual stimuli. Journal of Experimental Child Psychology, 11, 244-250.

Farthing, G. W., \& Opuda, M. J. (1974). Transfer of matching-to-sample in pigeons. Journal of the Experimental Analysis of Behavior, 21, 199-213.

Ginsburg, N. (1957). Matching in pigeons. Journal of Comparative Physiology and Psychology, 50, 261-263.

Herman, L. M., Hovancik, J. R., Gory, J. D., \& Bradshaw, G. L. (1989). Generalization of visual matching by a bottlenosed dolphin (Tursiops truncatus): Evidence for invariance of cognitive performance with visual and auditory materials. Journal of Experimental Psychology: Animal Behavior Processes, 15, 124.

Hochmann, J. R., Mody, S., \& Carey, S. (2016). Infants' representations of same and different in match-and non-match-to-sample. Cognitive Psychology, 86, 87-111.

Kastak, D., \& Schusterman, R. J. (1994). Transfer of visual identity matching-to-sample in two California sea lions (Zalophus californianus). Animal Learning \& Behavior, 22, 427-435.

Katz, J. S., Bodily, K. D., \& Wright, A. A. (2008). Learning strategies in matching to sample: If-then and configural learning by pigeons. Behavioural Processes, 77, 223-230. doi:10.1016/j.beproc.2007. 10.011

Katz, J. S., \& Wright, A. A. (2006). Same/different abstract-concept learning by pigeons. Journal of Experimental Psychology: Animal Behavior Processes, 32, 80-86. doi:10.1037/0097-7403.32.1.80

Katz, J. S., Wright, A. A., \& Bachevalier, J. (2002). Mechanisms of samedifferent abstract-concept learning by rhesus monkeys (Macaca mulatta). Journal of Experimental Psychology: Animal Behavior Processes, 28, 358-368. doi:10.1037//0097-7403.28.4.358

Magnotti, J. F., Katz, J. S., Wright, A. A., \& Kelly, D. M. (2015). Superior abstract-concept learning by Clark's nutcrackers (Nucifraga columbiana). Biology Letters, 11(5). doi:10.1098/rsbl.2015.0148

Mishkin, M., \& Delacour, J. (1975). An analysis of short-term visual memory in the monkey. Journal of Experimental Psychology: Animal Behavior Processes, 1, 326.

Pascalis, O., de Haan, M., \& Nelson, C. A. (2002). Is face processing species-specific during the first year of life? Science, 296(5571), $1321-1323$.

Pepperberg, I. M. (1987). Acquisition of the same/different concept by an African grey parrot (Psittacus erithacus): Learning with respect to categories of color, shape, and material. Animal Learning and Behavior, 15, 423-432.

Premack, D. (1978). On the abstractness of human concepts: Why it would be difficult to talk to a pigeon. In S. H. Hulse, H. Fowler, \& W. K. Honig (Eds.), Cognitive processes in animal behavior (pp. 423-451). Hillsdale, NJ: Erlbaum.

Premack, D. (1983). The codes of man and beasts. Behavioral Brain Science, 6, 125-167.

Schmidtke, K. A., Katz, J. S., \& Wright, A. A. (2010). Differential outcomes facilitate same/different concept learning. Animal Cognition, 13, 583-589. doi:10.1007/s10071-009-0292-2

Thompson, R. K. R. (1995). Natural and relational concepts in animals. In H. Roitblat \& J. A. Meyer (Eds.), Comparative approaches to cognitive science (pp. 175-224). Cambridge, MA: MIT Press.

Wasserman, E. A., DeVolder, C. L., \& Coppage, D. J. (1992). Nonsimilarity based conceptualization in pigeons via secondary or mediated generalization. Psychological Science, 3(6), 374-379.

Wilson, B., Mackintosh, N. J., \& Boakes, R. A. (1985). Matching and oddity learning in the pigeon: Transfer effects and the absence of 
relational learning. Journal of Experimental Psychology, 37, 295311. doi:10.1080/14640748508401172

Wright, A. A. (1997). Concept learning and learning strategies. Psychological Science, 8, 119-123. doi:10.1111/j.1467-9280.1997. tb00693.x

Wright, A. A., \& Delius, J. D. (2005). Learning processes in matching and oddity: The oddity preference effect and sample reinforcement. Journal of Experimental Psychology: Animal Behavior Processes, 31, 425-432. doi:10.1037/0097-7403.31.4.425

Wright, A. A., \& Katz, J. S. (2006). Mechanisms of same/different concept learning in primates and avians. Behavioural Processes, 72(3), 234-254. doi:10.1016/j.beproc.2006.03.009

Wright, A. A., \& Katz, J. S. (2007). Generalization hypothesis of abstractconcept learning: Learning strategies and related issues in Macaca mulatta, Cebus apella, and Columba livia. Journal of Comparative Psychology, 121, 387-397. doi:10.1037/0735-7036.121.4.387

Wright, A. A., Rivera, J. J., Katz, J. S., \& Bachevalier, J. (2003). Abstractconcept learning and list-memory processing by capuchin and rhesus monkeys. Journal of Experimental Psychology: Animal Behavior Processes, 29, 184-198.

Zentall, T. R., Edwards, C. A., Moore, B. S., \& Hogan, D. E. (1981). Identity: The basis for both matching and oddity learning in pigeons. Journal of Experimental Psychology: Animal Behavior Processes, 7, 70-86.

Zentall, T., \& Hogan, D. (1974). Abstract concept learning in the pigeon. Journal of Experimental Psychology, 102, 393-398. doi:10.1037/ h0035970 